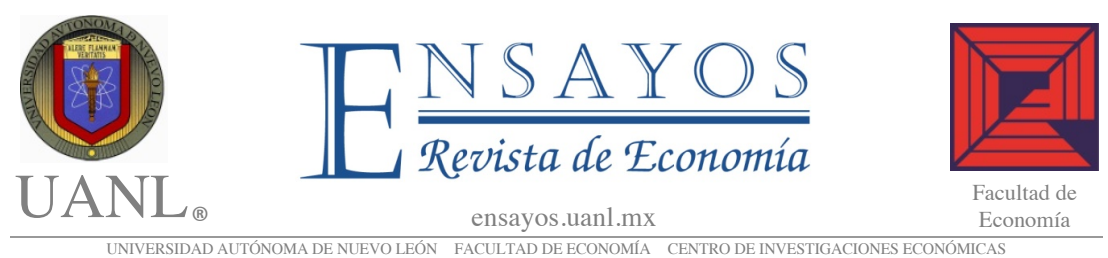

\title{
Cambio tecnológico y eficiencia logística del transporte de carga internacional a través del modelo DEA
}

\section{Technological change and efficiency of the logistics system of international freight through the DEA model}

\section{América Ivonne Zamora Torres}

\begin{tabular}{|c|c|}
\hline $\begin{array}{l}\text { Información del } \\
\text { artículo }\end{array}$ & Resumen \\
\hline $\begin{array}{l}\text { Recibido: } \\
27 \text { marzo } 2018\end{array}$ & $\begin{array}{l}\text { El presente estudio tiene como objetivo determinar la } \\
\text { eficiencia, el índice de Malmquist y cambio } \\
\text { tecnológico del sistema de transporte carretero de carga }\end{array}$ \\
\hline Aceptado: & internacional por entidad federativa, considerando los \\
\hline 12 junio 2019 & factores que afectan la movilización de carga \\
\hline $\begin{array}{l}\text { Clasificación JEL: } \\
\text { F10;F14; R40; R4L }\end{array}$ & $\begin{array}{l}\text { internacional, a través del modelo DEA y el índice } \\
\text { Malmquist. Se hace un análisis detallado del período } \\
2010 \text { a 2014, así como sobre los diferentes tipos de }\end{array}$ \\
\hline Palabras clave: & eficiencia: eficiencia técnica global, eficiencia técnica \\
\hline $\begin{array}{l}\text { Eficiencia; transporte } \\
\text { carretero de carga } \\
\text { internacional; DEA; } \\
\text { Malmquist }\end{array}$ & $\begin{array}{l}\text { y eficiencia de escala. Los resultados muestran un } \\
\text { aumento de la productividad total de los factores, sin } \\
\text { embargo ese incremento de productividad es } \\
\text { significativamente desigual entre las diferentes } \\
\text { entidades federativas. }\end{array}$ \\
\hline
\end{tabular}

* Secretaría Académica del Instituto de Investigaciones Económicas y Empresariales, Directora del centro de Estudios APEC y profesora investigadora. Universidad Michoacana de San Nicolás de Hidalgo. Email: americazt@hotmail.com

ISSN Electrónico: 2448-8402 | ISSN Impreso: 1870-221X | (C2019 Los autores @) () 


\begin{tabular}{|c|c|}
\hline Article information & Abstract \\
\hline Received: & This paper aims to determine the efficiency, the \\
\hline 27 march 2018 & Malmquist index and the technological change of the \\
\hline Accepted: & international trade, taking into consideration the factors \\
\hline 12 june 2019 & that affect the mobilization of goods through the DEA \\
\hline JEL Classification: & model and the Malmquist index. Performing a detailed \\
\hline F10; F14; R40; R4L & $\begin{array}{l}\text { analysis of the period } 2010 \text { to } 2014 \text { as well as the } \\
\text { different types of efficiency (overall technical }\end{array}$ \\
\hline Keywords: & efficiency, technical efficiency and efficiency of scale). \\
\hline Efficiency; & The results show an increase in the total productivity of \\
\hline international freight & the factors, however this increase of the productivity is \\
\hline transport; DEA; & significantly unequal between the different federative \\
\hline Malmquist & entities. \\
\hline
\end{tabular}

\section{Introducción}

La eficiencia en la logística del comercio internacional es parte importante en la competitividad que un país pueda tener, en el nivel mundial. Dicho sistema logístico se compone de cada una de las fases o etapas por las que el producto terminado debe pasar a fin de llegar al importador, ya sea consumidor final o intermediario. Por lo que, a grandes rasgos, el sistema logístico se puede agrupar, en: aduanas, almacenes, transporte internacional de carga y servicios logísticos (como trazabilidad y rastreo). Dentro de estos eslabones, uno de los pasos cruciales es sin duda el transporte internacional de carga, que habrá de transportar la mercancía cruzando fronteras no solo ejecutando en tiempo sino, además, preservando el producto en las mejores condiciones posibles.

En México, el transporte de carga terrestre es de suma importancia para el sector productivo nacional; ya que, actualmente, la red carretera nacional se ha convertido en la vía más relevante en México, para el movimiento de mercancías, dado que representan más del $60 \%$ de las exportaciones. Debido a ello, cabe preguntarse: ¿qué tan eficiente es el transporte carretero de carga internacional?

En este sentido, la infraestructura del transporte tiene un papel trascendental en el marco del comercio internacional; esto se da principalmente por el suministro de materias primas al sector industrial, al movimiento de productos intermedios a centros de procesamiento y a la distribución de productos terminados de los centros de almacenamiento al consumidor final, ya sea nacional o extranjero. Las características propias de los medios de transporte, como: cobertura, capacidad de tonelaje movido, distancia recorrida, tiempo de traslado y costos de operación, son las ventajas y desventajas por las que el usuario decide el reparto modal de los flujos en autotransporte. 
Para lo cual, las carreteras son fundamentales para el comercio, puesto que posibilitan el desplazamiento de mercancías de un mercado a otro de manera rápida en distancias cortas y medias. Dependiendo de su cobertura, brindan mayor flexibilidad que otros modos de transporte, y facilitan el movimiento de mercancías a través de diferentes medios de transporte, que generan redes de corredores intermodales de transporte, necesarios para el flujo expedito de las mercancías. Eso permite, a través del transporte multimodal, la facilitación de mercancías gracias al uso de unidades de carga estandarizada como contenedores o remolques (Puettman y Stadtler, 2010; Crainic y Florian, 2008).

Consecuentemente, una adecuada infraestructura del sistema de transporte de carga que esté a la vanguardia tecnológica y permita una mayor cobertura geográfica, así como una reducción en tiempos y costos, además de garantizar la entrega de los productos a comerciar en óptimas condiciones, puede ser la diferencia para que un producto se considere competitivo o incluso, propicie la mejora del crecimiento comercial internacional de un país.

A partir de los planteamientos presentados, surge la hipótesis de investigación siguiente: el transporte carretero de carga internacional en el comercio exterior muestra un incremento en su eficiencia durante del período 2010 a 2014, derivado de los cambios tecnológicos en el sector. De ahí que el objetivo de esta investigación sea determinar la eficiencia, el índice de Malmquist y el cambio tecnológico del sistema de transporte carretero de carga internacional, por entidad federativa.

\section{El transporte de carga y la logística del comercio internacional}

En los últimos años, la posibilidad de realizar diferentes análisis sobre esta temática se ha visto reforzada con la publicación del Índice de Desempeño Logístico, el cual ha aportado información valiosa sobre la situación de cada uno de los países. Este índice permite, entre otras cosas, establecer comparaciones entre las naciones e identificar aquellas áreas donde la deficiente logística puede suponer una limitación para su desarrollo económico (Banco Mundial, 2016).

Hertel y Mirza (2009) evalúan el efecto del índice sobre los flujos de comercio con datos de 95 países para el año 2001, obteniendo como resultado que el coeficiente del exportador pesa más que el del importador y los componentes aduanas e infraestructuras son los de mayor impacto sobre el comercio. También, Felipe y Kumar (2010) hacen un análisis por componentes del mismo índice, donde sus resultados muestran que la infraestructura es el factor 
determinante de los flujos comerciales asiáticos, seguido de la logística y las aduanas.

Acorde con el ranking de logística y comercio global realizado por el Banco Mundial (BM), la eficiencia de México en 2016 bajó a la posición 54, mientras que en 2014, en exportaciones e importaciones, se ubicó en la posición 50 de 160 economías, cuando en 2012 su posición era la 47. Esto refleja retroceso continuo en la competitividad logística de México en el comercio internacional.

Por otra parte, el Foro Económico Mundial llega a conclusiones similares al establecer que México se ubica en el lugar 68 de 144 en el Índice de Competitividad e Infraestructura.

Mankiw (2010) afirmó que, el comercio internacional es un acelerador del desarrollo. Según Hummels, Ishi y Yi (2001), el crecimiento del comercio se dio en virtud a una creciente especialización vertical entre países, por lo que un mayor número de transacciones de exportación e importación fueron hechas por cada producto final.

Acorde con FTA (2016), la logística tiene un rol vital en el bienestar de la sociedad, ya que sin la conectividad necesaria y el buen desempeño de la red logística de transporte, tanto la disponibilidad como la cantidad de los recursos con los que contamos, disminuiría drásticamente.

Según Manheim (1979), la mayoría de las actividades globales de transporte se llevan a cabo en cinco grandes modalidades: carretera, ferroviario, aéreo, acuático y ductos. Cada una de ellas se divide en dos o más medios específicos, y se evalúan en términos de los siguientes atributos:

- Ubicación: Grado de accesibilidad al sistema, facilidad de rutas.

- Movilidad: Cantidad de tránsito, capacidad y rapidez para transportar.

- Eficiencia: Relación entre costos totales (directos más indirectos) del transporte y su productividad.

La infraestructura de un país tiene un papel importante en la movilización de su comercio, tema que es ampliamente tratado en diversos estudios. Por ejemplo, Flitsch y Brümmerstedt (2015b) muestran en su estudio la importancia de las redes de transporte, mientras que Bougheas (1999), muestra las circunstancias en las que la infraestructura de transporte, afecta el volumen de comercio entre dos países.

Para Francois y Manchin (2007), el transporte y la infraestructura de comunicaciones, así como, la calidad de las instituciones son factores determinantes cuya importancia no sólo afecta a los niveles de exportaciones 
sino también a su probabilidad de referencia de entrega de bienes, con otros países; y, de esta manera, reflejan el estado de la infraestructura propia y de los socios comerciales.

Se considera que la reducción en los costos del comercio internacional es un tema importante, que se relaciona con las medidas de facilitación del comercio, el desarrollo económico y el crecimiento del comercio internacional.

Hummels (2007) sostuvo que el crecimiento del comercio internacional está positivamente relacionado con la reducción de los costos del transporte internacional. Por su parte, Brümmerstedt, Flitsch y Jahn (2015) muestran en su estudio funciones de costos de los modelos de carga, concluyendo sobre la gran importancia que tiene el transporte carretero en el comercio internacional intermodal.

Según Sourdin y Pomfret (2012), cuando se asocia la liberalización del comercio con reducciones de costos de transporte debido al uso de contenedores, mejores aviones y logística, se da lugar a una notable expansión del comercio internacional. Por lo que se puede afirmar que el transporte resulta ser una actividad de alto valor agregado, dentro de una cadena de suministro, donde hasta dos tercios de los costos logísticos se pueden asociar con el transporte de bienes (Caplice y Sheriff, 2011) y (Federal Highway Administration, 2005).

Derivado de lo expuesto en este apartado, permea la necesidad de un eficiente sistema de transporte que incluya la modernización de los factores o variables del sistema de transporte carretero, como pueden ser: mejoras en infraestructura, tanto carretera como del medio de transporte, capacitaciones, implementación de sistemas de calidad que impliquen una reducción de costos $\mathrm{y}$, por ende, un incremento en el comercio internacional y su eficiencia.

\section{Eficiencia técnica y cambio tecnológico}

Las ideas de Farrell (1957) han sido aplicadas a través de dos metodologías: la estimación de fronteras estocásticas y las mediciones DEA. El Análisis de la Envolvente de Datos (DEA) es una técnica utilizada para la medición de la eficiencia comparativa de unidades homogéneas. Partiendo de los inputs y outputs, este método proporciona un ordenamiento de los agentes o DMU (Unidad de Toma de Decisión), otorgándoles una puntuación de eficiencia relativa. Los modelos DEA aprovechan el know-how de las DMU y una vez determinado quién es eficiente, buscan fijar objetivos de mejora para las DMUs no eficientes, a partir de los logros de los primeros. Los modelos DEA 
pueden tener dos orientaciones, hacia la optimización en la combinación de inputs o hacia la optimización en la producción de outputs (Cooper, Seiford \& Tone, 2007).

La habilidad de obtener el máximo producto, dados ciertos insumos y tecnología fija, se define como eficiencia técnica. Las medidas de eficiencia técnica, en el análisis de frontera determinística o estocástica, están inspiradas en las propuestas de Debreu (1951) y Farrell (1957). Se puede decir que las DMUs que operan en la frontera de producción, son técnicamente eficientes.

La medida de eficiencia técnica Debreu-Farrell se define como la máxima contracción equi-proporcional en todos los insumos, que permite continuar con la producción. Es una medida de carácter radial. Si no es posible contraer de forma equi-proporcional todos los insumos, se dice que el vector de insumos es técnicamente eficiente.

\subsection{Cambio tecnológico y eficiencia técnica variable en el tiempo}

La eficiencia técnica cambiante en el tiempo, se refiere al acercamiento o distanciamiento del nivel de producción de una unidad productiva, con respecto a la frontera de posibilidades de producción del sector a la que pertenece, respecto de diferentes períodos de tiempo; mientras que el cambio técnico o cambio tecnológico, se asocia con un desplazamiento de la frontera de posibilidades de producción. Al disponer de información de datos panel, que involucre varios períodos, es posible que se presente cambio técnico, así como también que la eficiencia técnica cambie con el tiempo.

Sin duda, el artículo de Solow (1957) sobre el cambio tecnológico y la función de producción agregada permitieron que recobrara fuerza el concepto de productividad total de los factores (PTF). En dicho artículo, Solow cuantifica las variaciones de la producción debidas al progreso técnico en forma residual, razón por la cual al progreso técnico se lo conoce también como residuo de Solow o PTF.

\section{Metodología}

La eficiencia medida como el grado mínimo de recursos que se utilizan, asociado a un máximo nivel de outputs generados, logra una optimalidad impuesta bajo ciertas condiciones como lo son, los precios y la tecnología. De este tipo de análisis, se desprende una frontera o limite derivado de las observaciones realizadas para cada una de las unidades consideradas, que pueden ser empresas, instituciones, países, regiones, etc. 
Cuando dicho óptimo está definido por la función de producción, a la medida de eficiencia que se obtiene se le denomina eficiencia técnica. Mientras que, si la comparación se realiza considerando un óptimo de índole económica (como pueden ser costos, maximización de ingresos o beneficios), la medida de eficiencia que resulta de aplicar el modelo, se denomina eficiencia económica.

El primero en utilizar la idea de Malmquist, para la comparación de una institución en dos momentos diferentes de tiempo dado, fue Moorteen en 1961, cuando buscaba ver cómo un input puede ser deflactado para producir el nivel observado de output del otro período.

Caves, Christensen y Diewert, en 1982, establecieron la relación entre los índices de Malmquist (1953) y Törnqvist (1936) desarrollando el índice de Malmquist, a través de dos enfoques; uno, que analiza las diferencias de productividad como las diferencias en el máximo output alcanzable, dado cierto nivel de inputs conocido como índice de Malmquist de productividad, basado en el output; y el segundo, analiza las diferencias de productividad como las diferencias en el mínimo nivel de inputs que permite producir ciertos niveles de outputs determinados, conocido como índice de Malmquist de productividad, basado en el input.

Berg, Forsund y Jansen (1992) hicieron la conexión entre los conceptos de función de distancia y las medidas de eficiencia de Farrell (1957); a partir de ahí, se pudieron observar las unidades analizadas ineficientes, sustituyendo el concepto de frontera tecnológica por tecnología.

El modelo a utilizar en el presente trabajo es el modelo de índice de Malmquist de productividad, que está basado en el input la justificación, y que parte de que el máximo beneficio tendría que ser un mayor flujo comercial o, dicho de otro modo, una mayor entrada y salida de productos en las diferentes entidades federativas, por lo que no cabría esperar una reducción o un output estable, sino por el contrario una minimización de los recursos empleados por el gobierno y empresas, que se traduciría teóricamente en un aumento de recursos disponibles en otros sectores, que también coadyuven al crecimiento económico del país.

Adicionalmente, acorde con Lovell (2002), a pesar de que la exogeniedad no es un problema estadístico en DEA, en el sentido que lo es para los modelos econométricos, la elección entre las medias orientadas en inputs o en outputs se someten a las mismas consideraciones. Por lo que el sistema de transporte carretero de carga internacional de las diferentes entidades federativas, objeto 
de estudio, están sometidas a condiciones de demanda, ajustando libremente sus inputs, así, el modelo orientado a los inputs sería el más apropiado.

Para ilustrar el cálculo del índice de Malmquist, supóngase que la función de trasformación que describe la tecnología de las empresas, en cada período, es:

$F_{t}\left(Y^{t}, X^{t}\right)=0 \quad t=1, \ldots, T$

Donde $y^{t}=\left(y_{1_{1} t}^{t}, \ldots, y_{N}^{t}\right) \in R_{N}^{+}$es el vector de outputs y $x^{t}=\left(x_{1}^{t}, \ldots, x_{M}^{t}\right) \in$ $R_{M}^{+}$denota el vector de inputs, correspondientes ambos al período $t$.

La tecnología puede ser representada de una forma más conveniente a través de la "función distancia de input", utilizada por Caves et al. (1982):

$$
D^{r}\left(y^{s}, x^{s}\right)=\operatorname{Max} \mu_{r s}\left[\mu_{r s}: F_{r}\left(y^{s}, \frac{x^{s}}{\mu_{r s}}\right)=0\right] \quad r, s=1, \ldots, T ; \quad r<s
$$

En donde, el escalar $\mu_{r s}$ es la máxima deflación del vector de inputs del período $\mathrm{s}\left(x^{s}\right)$, tal que el vector inputs deflactado resultante $x^{s} / \mu_{r s}$ y el vector de outputs $\left(y^{S}\right)$ estén en la frontera del período r. si $\mathrm{r}=\mathrm{s}$ se está comprando cada empresa con la frontera del período al que pertenece, por lo que la función distancia de input $D^{r}\left(y^{r}, x^{r}\right) \geq 1$, siendo igual a la unidad en el caso de que la empresa evaluada sea eficiente y, por tanto, se encuentre en la frontera. Por el contrario, si $\mathrm{r} \neq s$ la función distancia puede tomar valores inferiores a la unidad, ya que la observación pertenece a un período diferente del de la frontera, con la cual se está comparando (frontera de referencia).

El índice de Malmquist de productividad basado en los inputs, tomando la tecnología del período $r$ como referencia, se define, como:

$$
M_{r}\left(y^{s}, x^{S}, y^{r}, x^{r}\right)=\frac{D^{r}\left(y^{r}, x^{r}\right)}{D^{r}\left(y^{s}, x^{s}\right)}
$$

Un $M_{r}>1$ indica que la productividad del período $s$ es superior a la del período $r$, puesto que la deflación necesaria del vector de inputs del período $r$, para estar en la frontera del período $\mathrm{r}$, es superior a la aplicable al vector de inputs del período s para que esté en la frontera del período r. Por el contrario, un $M_{r}$ índica que la productividad ha descendido entre los períodos $\mathrm{r}$ y $s$.

Los números índices que frecuentemente se han utilizado para analizar el cambio productivo, son: el índice de Fisher (1922), el índice de Törnqvist (1936) y el índice de Malmquist (1953). Las ventajas de la utilización de los índices de Fisher y Törnqvist es que pueden ser calculados sin recurrir a la 
estimación de la tecnología subyacente, si no que únicamente precisan datos de cantidades (de outputs o de inputs) y de precios.

Sin embargo, como señala Grifell et al. (1993a), el índice de Malmquist presenta tres ventajas frente al de Fisher y Törnqvist. En primer lugar, no necesita suponer comportamiento minimizador de costes o maximizador de ingresos. En segundo lugar, no precisa de datos relativos o precios, lo cual es una gran ventaja, sobre todo en aquellos casos en los que existan graves carencias estadísticas, o simplemente en los casos en los que la existencia de regulaciones sobre los mismos y/o presencia de poder de mercado, reflejado en los precios, hagan desaconsejable su utilización. Por último, permite la descomposición del cambio productivo, cambio en la eficiencia técnica (catching-up) y cambio técnico (o desplazamiento de la frontera), siendo este el objetivo central del presente trabajo. El inconveniente principal que presenta el índice Malmquist es que, para su cálculo individual, precisa el previo cálculo de la distancia, por lo que requiere la estimación de la función de producción.

La descomposición del cambio productivo en cambio en la eficiencia técnica y progreso (regreso) técnico fue una cuestión abordada por Nishimizu y Page (1982). Estos autores analizaron el sector industrial de la antigua Yugoslavia en el período 1965-78 mediante la especificación, y posterior estimación, por métodos de programación matemática, de una función de producción translong imponiendo rendimientos constantes a escala.

Después de este pionero trabajo, Berg et al. (1992), obtienen una similar descomposición del cambio productivo utilizando el índice de Malmquist. Para su estimación, emplean la técnica no paramétrica determinista DEA, mucho más flexible que la técnica paramétrica empleada por Nishimizu et al. (1982).

Desde entonces, las aportaciones empíricas y teóricas más relevantes de la referida metodología corresponden a los trabajos de Grifell et al. (1993a), que aplican la metodología de Berg et al. (1992), para analizar el cambio productivo de las cajas de ahorro españolas, y la de Grifell et al. (1993b), en donde se propone una descomposición alternativa que permite analizar adicionalmente la posible presencia de sesgo tecnológico, y, por último, la de Grifell et al. (1996), en donde se demuestra que el índice de Malmquist ofrece una medida imprecisa del cambio productivo, cuando los rendimientos a escala no son constantes.

La referida descomposición del índice de Malmquist en el efecto catching-up y desplazamiento de la frontera puede expresarse como (Berg, Forsund, \& Jansen, 1992), (Grifell \& Lovell, Grifell, Deregulation and Productivity 
Decline: The case of Spanish Saving Banks. , 1993a), (Grifell \& Lovell, 1993b) y (Grifell \& Lovell, 1996) :

$$
M_{r}\left(y^{s}, x^{s}, y^{r}, x^{r}\right)=\frac{D^{r}\left(y^{r}, x^{r}\right)}{D^{r}\left(y^{s}, x^{s}\right)}=\frac{D^{r}\left(y^{r}, x^{r}\right)}{D^{r}\left(y^{s}, x^{s}\right)} \cdot \frac{D^{s}\left(y^{s}, x^{s}\right)}{D^{r}\left(y^{s}, x^{s}\right)}
$$

El primer cociente representa el acercamiento de las empresas a la frontera, ocurrido entre los períodos $\mathrm{r}$ y s, mientras que el segundo término muestra el desplazamiento relativo de la frontera entre los dos períodos.

Si la empresa se encuentra en ambos períodos en sus fronteras respectivas, el primer término será igual a 1 y el cambio productivo experimentado entre los dos períodos vendrá explicado únicamente por el movimiento de la frontera. Por el contrario, si el segundo término es 1 (la frontera no se ha desplazado), los cambios de productividad estimados por $M_{r}$ vendrán explicados únicamente por los cambios en la eficiencia de las empresas en ambos períodos (catching-up). En los demás casos, los cambios productivos reflejados en $M_{r}$ serán una mezcla de cambios en la eficiencia y desplazamientos de la frontera.

Färe y Lovell (1978) formalizaron la relación existente entre la función distancia de input y las medidas de Farell ahorradoras de inputs $E_{r_{r}}\left(y^{r}, x^{r}\right)$, y demostraron que la función distancia es igual a la inversa de la medida de Farell ahorradora de inputs $D^{r}\left(y^{r}, x^{r}\right)=\left[E_{r_{r}}\left(y^{r}, x^{r}\right)\right]^{-1}$

Dado que en el caso de rendimientos constantes a escala, se cumple que $\frac{x_{r}^{s}}{x_{r^{r}}}=$ $y^{s} / y^{r}$, el índice de Malmquist puede ser escrito en este caso, como:

$$
M_{r}\left(y^{s}, x^{s}, y^{r}, x^{r}\right)=\frac{D^{r}\left(y^{r}, x^{r}\right)}{D^{r}\left(y^{s}, x^{s}\right)}=\frac{E_{r s}}{E_{r r}}=\frac{\frac{x_{r}^{s}}{x_{s}^{r}}}{x_{r}}=\frac{\frac{y^{s}}{x^{s}}}{y^{r}}
$$

Que en este caso, se reduce a un simple ratio de índices de productividad de los períodos $\mathrm{r}$ y $\mathrm{s}$.

Para este sencillo ejemplo, la descomposición del índice de Malmquist en el efecto catching-up (CU) y el cambio técnico o desplazamiento de la frontera (DF) puede expresarse, como:

$$
M_{r}\left(y^{s}, x^{s}, y^{r}, x^{r}\right)=\frac{E_{r s}}{E_{r r}}=\frac{E_{s s}}{E_{r r}} \cdot \frac{E_{r s}}{E_{s s}}=\mathrm{CU}\left(y^{s}, x^{s}, y^{r}, x^{r}\right) . \mathrm{DF}\left(y^{s}, x^{s}, y^{r}, x^{r}\right)
$$

En donde, el catching-up o acercamiento relativo a la frontera, ocurrido entre el período $r$ y $s$, sería $\left.\mathrm{CU}\left(y^{s}, x^{s}, y^{r}, x^{r}\right)=\frac{E_{S S}}{E_{r r}}=\left(x_{s}^{s} / x_{s}\right) / x_{r}^{r} / x^{r}\right)^{8}$ y el 
desplazamiento de la frontera entre los dos períodos vendría expresado por, $\operatorname{DF}\left(y^{s}, x^{s}, y^{r}, x^{r}\right)=\frac{E_{r s}}{E_{s s}}==\left(x_{r}^{s} / x^{s}\right) /\left(x_{s}^{s} / x^{s}\right)=x_{r}^{s} / x_{s}^{s}$.

En cuanto a las especificaciones, se considera un modelo con orientación output en el cálculo del índice Malmquist, ya que se pretende maximizar las exportaciones con los insumos que se tienen.

Otra de las limitantes de los modelos DEA es el número total de inputs y outputs que se pueden utilizar para el análisis. Específicamente, es recomendable que el número de DMUS a ser analizadas (en la presente investigación entidades federativas) sea al menos tres veces más grande que el número en suma de inputs y outputs incluidos en el análisis (Cooper, Seiford, $\&$ Tone, 2006). Esta limitante implica de entrada un problema, al tratar de seleccionar los inputs y outputs más relevantes, para lo cual se consultó información de los reportes de la Organización Mundial de Comercio (OMC) y de SCCP principalmente, de donde se seleccionarán los siguientes inputs y outputs:

\section{Inputs:}

Los inputs seleccionados se consideraron tomando en cuenta cuatro variables principales a) la infraestructura carretera; b) la infraestructura de las empresas transportistas; c) recursos humanos y d) calidad. De dichas variables se desprenden los inputs a utilizar, considerando el cambio tecnológico en la infraestructura carretera de las empresas transportistas y estándares de calidad, así como capacitación en los recursos humanos. Quedando la operacionalización de las variables, de la siguiente manera:

a) Infraestructura carretera. La infraestructura carretera se medirá a través de dos indicadores:

1. Las carreteras pavimentadas, es decir, el número de kilómetros de carretera que al momento del estudio se registren como asfaltados. Cabe señalar que acorde con las regulaciones internacionales, la circulación de vehículos de carga internacional solo puede transitar por este tipo de caminos.

2. Densidad vehicular de carga. Este indicador se refiere al número de vehículos de carga que efectivamente circulan por las carreteras y autopistas, con dicho indicador se puede medir el aprovechamiento de las carreteras.

b) Infraestructura de empresas transportistas. Otra variable de infraestructura es la flota con la que cuentan las empresas transportistas de carga dedicadas al 
comercio internacional, dicha infraestructura -para efectos de este estudio- se ha dividido en tres indicadores.

1. Unidades motrices de carga. Corresponde al número de vehículos en uso para carga de transporte internacional.

2. Número de empresas de transporte internacional. Si bien actualmente existe en la actualidad un vasto número de empresas dedicadas al movimiento de bienes, solo algunas cuentan realmente con los registros y requerimientos para realizar transporte de bienes, de manera internacional; de ahí, la importancia de conocer cuál es el verdadero recurso con el que se cuenta para el movimiento de mercancías.

3. Licencias internacionales. Este indicador permite medir el número de choferes que cuentan con una licencia para conducir afuera de su territorio nacional transporte de carga, actualmente, tener dicha licencia es requisito indispensable en el tránsito de mercancías.

c) Recursos humanos. Esta variable pretende medir el número de recursos humanos con los que se cuenta, a fin de que estén a disposición para hacer más eficiente la circulación de mercancía.

1. Conductores capacitados. Este indicador mide el número de conductores que han tomado los cursos correspondientes y han pasado las evaluaciones que les permiten ser conductores certificados para mover mercancías internacionalmente.

d) Calidad. La calidad de los servicios logísticos del transporte es una variable que se considera crucial en términos de eficiencia, y los indicadores son:

1. Centros de capacitación. Dicho indicador mide el número de centros de capacitación que existen en el país, para servicios especializados de carga de transporte internacional.

2. Número de inspecciones realizadas. Este indicador se refiere al número de revisiones que se realizan tanto de la carga como del transporte y el transportista, a fin de vigilar que todo esté en forma y no se cometan ilícitos.

3. Número de verificaciones. El indicador de verificaciones abarca las supervisiones de control de calidad, cumplimiento de regulaciones y certificaciones.

\section{Outputs:}

Por su parte, la selección de outputs obedece al fin último de las operaciones de comercio exterior: un mayor flujo de productos en los mercados internacionales, por lo que será importante medir el nivel de comercio internacional que se tiene, particularmente, a través de las exportaciones, considerando el valor de la mercancía comercializada, donde el indicador a utilizar, es: 
valor de las exportaciones. Este indicador considera el valor registrado de la mercancía en aduana, dicho valor está estimado en dólares americanos y considera un valor $\mathrm{FOB}^{1}$.

\section{Resultados}

El índice de productividad de Malmquist genera una frontera de posibilidades de producción para cada período, en este caso, 2010 y 2014, a fin de analizar los cambios en la productividad de cada una de las DMUs en dichos períodos. La medición del índice de Malmquist representa el crecimiento de la productividad total de los factores de una unidad de toma de decisiones, particularmente del sistema de transporte de carga por entidad federativa, en donde se refleja el progreso o retroceso en la eficiencia y, por otro lado, el progreso o retroceso de la frontera tecnológica entre dos períodos de tiempo bajo el marco de múltiples entradas y salidas (Cooper, Seiford \& Tone, 2007). De tal manera que, un coeficiente mayor a 1 representa un cambio o progreso en la productividad total de los factores de la unidad analizada, un valor igual a 1 representa un valor constante en la productividad y, finalmente, un valor inferior a 1 representa un deterioro en la productividad total de los factores. En análisis llevado a cabo se realizó a través del software MAXDEA.

Se observa, en la tabla 1, que durante el período estudiado en general la mayoría de las entidades federativas tuvieron un aumento en su productividad, en lo que se refiere al transporte de carga carretero, entre las entidades que sobresalen con el mayor incremento están: Durango en el período de 2012 a 2013, el Estado de México, San Luis Potosí, Sinaloa, Chihuahua y Guanajuato. Por su parte, entidades como Coahuila, Morelos y Colima son las entidades que mostraron un menor crecimiento en su productividad.

Cabe señalar que, para el período 2012 - 2013, el gobierno de Durango invirtió un monto de 2,826 millones de pesos en infraestructura carretera, donde sobresalen proyectos como los caminos: Durango-La Flor, La Flor-Mimbres, Durango-Parral, Durango-Tepic, entre otros; por su parte, los gobiernos de Chihuahua y Guanajuato incrementaron la inversión en infraestructura carretera durante los períodos 2012-2013 y 2013-2014, al mismo tiempo que se realizan innovaciones en el sistema carretero y de transporte de carga internacional.

\footnotetext{
${ }^{1}$ Free on Board o Libre a Bordo, INCOTERMS 2010.
} 
Tabla 1

Índice Malmquist del transporte carretero de carga 2010-2014

\begin{tabular}{lrrrrrr}
\hline \multicolumn{1}{c}{ DMU } & 2010-2011 & 2011-2012 & 2012-2013 & 2013-2014 & 2010-2014 & PROMEDIO \\
\hline Aguascalientes & 1.03 & 1 & 1 & 1 & 1.09 & 1.02 \\
Baja California & 0.94 & 1.11 & 1.08 & 1.08 & 0.35 & 0.91 \\
Baja California & & & & & & \\
Sur & 1 & 1 & 1 & 1 & 1.17 & 1.03 \\
Campeche & 1 & 1 & 1 & 1 & 0.73 & 0.95 \\
Chiapas & 0.88 & 1 & 1 & 1 & 1.01 & 0.98 \\
Chihuahua & 1.10 & 1.05 & 3.79 & 0.94 & 0.62 & 1.50 \\
Coahuila & 1.33 & 0.62 & 1.05 & 0.98 & 0.03 & 0.80 \\
Colima & 1 & 1 & 0.04 & 0.69 & 1.69 & 0.88 \\
Ciudad de México & 1 & 1 & 1 & 1.13 & 1.50 & 1.13 \\
Durango & 0.99 & 1.02 & 15.32 & 0.12 & 1.99 & 3.89 \\
Estado de México & 1.61 & 1.29 & 1 & 1.09 & 4.02 & 1.80 \\
Guanajuato & 1.61 & 0.57 & 0.95 & 2.88 & 1.38 & 1.48 \\
Guerrero & 1 & 1 & 1 & 1 & 0.59 & 0.92 \\
Hidalgo & 0.72 & 0.68 & 0.97 & 1.23 & 0.92 & 0.90 \\
Jalisco & 0.76 & 1.30 & 1.08 & 0.91 & 1.77 & 1.16 \\
Michoacán & 0.96 & 1.11 & 1.23 & 0.72 & 1.13 & 1.03 \\
Morelos & 1 & 1 & 1 & 1 & 0.05 & 0.81 \\
Nayarit & 1 & 1 & 1 & 1 & 1.10 & 1.02 \\
Nuevo León & 1.12 & 0.95 & 1.42 & 0.81 & 0.84 & 1.03 \\
Oaxaca & 1 & 1 & 1.69 & 1 & 0.67 & 1.07 \\
Puebla & 1.09 & 0.56 & 0.87 & 0.96 & 2.99 & 1.29 \\
Querétaro & 1.35 & 1.45 & 0.96 & 1.08 & 0.01 & 0.97 \\
Quintana Roo & 1 & 1 & 1 & 1 & 1.44 & 1.09 \\
San Luis Potosí & 1.08 & 1.19 & 1.12 & 1.12 & 4.05 & 1.71 \\
Sinaloa & 1.17 & 0.68 & 1.23 & 3.98 & 1.07 & 1.63 \\
Sonora & 0.95 & 1.01 & 1.30 & 0.94 & 0.43 & 0.93 \\
Tabasco & 1 & 1 & 0.94 & 0.79 & 1.16 & 0.98 \\
Tamaulipas & 1.04 & 1.00 & 1.02 & 1.20 & 1.22 & 1.10 \\
Tlaxcala & 1 & 1 & 1 & 1 & 1.04 & 1.01 \\
Veracruz & 1.22 & 1.51 & 0.84 & 0.96 & 0.14 & 0.93 \\
Yucatán & 0.726 & 0.860 & 1 & 1 & 1.07 & 0.93 \\
Zacatecas & 1 & 1 & 1 & 1 & 0.76 & 0.95 \\
\hline Funt elabo & & 1 & 1 & 1 &
\end{tabular}

Fuente: elaboración propia con base en los cálculos realizados a partir de la metodología DEA y Malmquist.

Por lo que, para el conjunto del sector logístico de transporte carretero de carga contemplado en este estudio, los niveles medios de cambio en eficiencia que se alcanzaron, muestran que la mayor parte de las entidades federativas no llevan el ritmo de crecimiento de las entidades federativas líderes, con respecto al sector de transporte carretero de carga internacional. Mientras que las evoluciones medias de productividad experimentadas, se deben al cambio técnico en el desplazamiento de la frontera. Ya que, el cambio tecnológico mayor lo presentó Ciudad de México siendo que, comparado con el más bajo (Baja California Sur), es un 39.1914 por ciento superior, lo cual denota un enorme diferencial entre las entidades. 
Tabla 2

Cambio Tecnológico 2010-2014

\begin{tabular}{|c|c|c|c|c|c|c|}
\hline DMU & 2010-2011 & 2011-2012 & $2012-2013$ & $2013-2014$ & $2010-2014$ & PROMEDIO \\
\hline Aguascalientes & 1.13 & 0.75 & 1.18 & 1.21 & 0.96 & 1.05 \\
\hline Baja California & 0.97 & 1.08 & 1.53 & 1.13 & 1.65 & 1.27 \\
\hline $\begin{array}{l}\text { Baja California } \\
\text { Sur }\end{array}$ & 1.25 & 0.12 & 0.93 & 0.45 & 0.47 & 0.64 \\
\hline Campeche & 1.02 & 1.07 & 1.27 & 0.67 & 1.17 & 1.04 \\
\hline Chiapas & 1.23 & 0.57 & 1 & 0.81 & 0.79 & 0.88 \\
\hline Chihuahua & 1.28 & 0.94 & 2.24 & 1.02 & 0.98 & 1.29 \\
\hline Coahuila & 1.38 & 0.65 & 1.16 & 0.84 & 0.94 & 0.99 \\
\hline Colima & 1.32 & 0.99 & 1.62 & 1.09 & 0.42 & 1.09 \\
\hline $\begin{array}{l}\text { Ciudad de } \\
\text { México }\end{array}$ & 1.26 & 0.9 & 2.22 & 6.07 & 18.42 & 5.77 \\
\hline Durango & 0.95 & 0.67 & 1.67 & 1.06 & 0.66 & 1.00 \\
\hline $\begin{array}{l}\text { Estado de } \\
\text { México }\end{array}$ & 1.67 & 0.65 & 1.05 & 1.35 & 1.99 & 1.34 \\
\hline Guanajuato & 1.33 & 0.39 & 1.48 & 1.07 & 0.94 & 1.04 \\
\hline Guerrero & 1.23 & 1.01 & 1.33 & 0.77 & 1.41 & 1.15 \\
\hline Hidalgo & 1.31 & 1.07 & 1.14 & 0.96 & 1.46 & 1.19 \\
\hline Jalisco & 1.35 & 0.71 & 1.35 & 0.84 & 1.05 & 1.06 \\
\hline Michoacán & 1.04 & 0.75 & 1.52 & 0.75 & 1.32 & 1.08 \\
\hline Morelos & 1.38 & 0.81 & 1.36 & 1.03 & 0.89 & 1.09 \\
\hline Nayarit & 1.25 & 1.15 & 1.05 & 0.71 & 0.19 & 0.87 \\
\hline Nuevo León & 1.12 & 0.89 & 1.41 & 0.81 & 1.1 & 1.07 \\
\hline Oaxaca & 1.47 & 0.36 & 1.63 & 0.93 & 0.8 & 1.04 \\
\hline Puebla & 1.72 & 0.6 & 1.21 & 0.79 & 1.05 & 1.07 \\
\hline Querétaro & 1.13 & 0.97 & 1.01 & 1 & 1.51 & 1.12 \\
\hline Quintana Roo & 3.27 & 0.1 & 1.56 & 1.06 & 0.36 & 1.27 \\
\hline San Luis Potosí & 1.23 & 0.9 & 1.27 & 0.79 & 1.14 & 1.07 \\
\hline Sinaloa & 1.13 & 0.65 & 1.19 & 1.04 & 0.9 & 0.98 \\
\hline Sonora & 1.28 & 1 & 1.17 & 0.71 & 1.41 & 1.11 \\
\hline Tabasco & 3.59 & 0.79 & 1.64 & 0.81 & 0.85 & 1.54 \\
\hline Tamaulipas & 1.21 & 0.96 & 1.65 & 1.22 & 2.31 & 1.47 \\
\hline Tlaxcala & 2.54 & 0.39 & 1.06 & 0.93 & 1.14 & 1.21 \\
\hline Veracruz & 1.24 & 0.56 & 1.62 & 0.97 & 0.99 & 1.08 \\
\hline Yucatán & 3.94 & 0.35 & 1.33 & 1.09 & 0.63 & 1.47 \\
\hline Zacatecas & 1.25 & 1.44 & 0.96 & 1.25 & 1.07 & 1.19 \\
\hline $\begin{array}{l}\text { Media } \\
\text { geométrica }\end{array}$ & 1.4 & 0.67 & 1.33 & 0.97 & 1.04 & 1.04 \\
\hline
\end{tabular}

Fuente: elaboración propia con base en los cálculos realizados a partir de la metodología DEA y Malmquist.

Al realizar el análisis del cambio en la eficiencia de manera desagregada, se puede observar el cambio en la eficiencia de escala, el cambio de la eficiencia técnica pura y la eficiencia técnica global, compuesta por las dos primeras, además del cambio tecnológico y el índice Malmquist del período estudiado $(2010-2014)$.

La tabla 3 muestra como el cambio tecnológico y el cambio en la eficiencia técnica global tuvieron un comportamiento opuesto, algunas entidades federativas presentaron progreso tecnológico, sobresaliendo el de Ciudad de 
México, Tamaulipas y el Estado de México. En cuanto al índice de Malmquist, los estados que mostraron un mayor incremento en la productividad del transporte carretero de carga internacional, para el período 2010 - 2014, fueron en orden descendente: Sinaloa, Guanajuato, Querétaro, Estado de México, Michoacán, Ciudad de México, Durango, San Luis Potosí, Guerrero, Tlaxcala, Campeche, Tamaulipas y Morelos.

Tabla 3

Productividad transporte carretero de carga internacional 2010-2014

\begin{tabular}{|c|c|c|c|c|c|}
\hline DMU & $\begin{array}{c}\text { Cambio } \\
\text { eficiencia } \\
\text { de escala }\end{array}$ & $\begin{array}{c}\text { Cambio } \\
\text { eficiencia } \\
\text { técnica } \\
\text { pura }\end{array}$ & $\begin{array}{c}\text { Cambio } \\
\text { eficiencia } \\
\text { técnica } \\
\text { global }\end{array}$ & $\begin{array}{c}\text { Cambio } \\
\text { tecnológico }\end{array}$ & $\begin{array}{c}\text { Índice } \\
\text { Malmquist }\end{array}$ \\
\hline Aguascalientes & 1 & 1 & 1 & 0.96 & 0.96 \\
\hline Baja California & 0.73 & 0.90 & 0.66 & 1.65 & 1.09 \\
\hline Baja California Sur & 0.74 & 1 & 0.74 & 0.47 & 0.35 \\
\hline Campeche & 1 & 1 & 1 & 1.17 & 1.17 \\
\hline Chiapas & 0.93 & 1 & 0.93 & 0.79 & 0.73 \\
\hline Chihuahua & 1.03 & 1 & 1.03 & 0.98 & 1.01 \\
\hline Coahuila & 0.66 & 1 & 0.66 & 0.94 & 0.62 \\
\hline Colima & 5.06 & 0.02 & 0.08 & 0.42 & 0.03 \\
\hline Ciudad de México & 1.00 & 0.09 & 0.09 & 18.42 & 1.69 \\
\hline Durango & 1.01 & 2.26 & 2.28 & 0.66 & 1.50 \\
\hline Estado de México & 1 & 1 & 1 & 1.99 & 1.99 \\
\hline Guanajuato & 1.45 & 2.97 & 4.30 & 0.94 & 4.02 \\
\hline Guerrero & 0.98 & 1 & 0.98 & 1.41 & 1.38 \\
\hline Hidalgo & 0.87 & 0.46 & 0.40 & 1.46 & 0.59 \\
\hline Jalisco & 0.96 & 0.91 & 0.88 & 1.05 & 0.92 \\
\hline Michoacán & 1.45 & 0.93 & 1.34 & 1.32 & 1.77 \\
\hline Morelos & 1.27 & 1 & 1.27 & 0.89 & 1.13 \\
\hline Nayarit & 0.27 & 1 & 0.27 & 0.19 & 0.05 \\
\hline Nuevo León & 1 & 1 & 1 & 1.10 & 1.10 \\
\hline Oaxaca & 1.04 & 1 & 1.04 & 0.80 & 0.84 \\
\hline Puebla & 0.80 & 0.80 & 0.64 & 1.05 & 0.67 \\
\hline Querétaro & 1.49 & 1.32 & 1.98 & 1.51 & 2.99 \\
\hline Quintana Roo & 0.04 & 1 & 0.04 & 0.36 & 0.01 \\
\hline San Luis Potosí & 0.86 & 1.48 & 1.27 & 1.14 & 1.44 \\
\hline Sinaloa & 0.98 & 4.60 & 4.50 & 0.90 & 4.05 \\
\hline Sonora & 0.79 & 0.97 & 0.76 & 1.41 & 1.07 \\
\hline Tabasco & 1.00 & 0.51 & 0.51 & 0.85 & 0.43 \\
\hline Tamaulipas & 0.53 & 0.96 & 0.50 & 2.31 & 1.16 \\
\hline Tlaxcala & 1.07 & 1 & 1.07 & 1.14 & 1.22 \\
\hline Veracruz & 0.80 & 1.32 & 1.06 & 0.99 & 1.04 \\
\hline Yucatán & 0.10 & 2.13 & 0.22 & 0.63 & 0.14 \\
\hline Zacatecas & 1 & 1 & 1 & 1.07 & 1.07 \\
\hline Media geométrica & 0.82 & 0.90 & 0.73 & 1.04 & 0.76 \\
\hline
\end{tabular}

Fuente: elaboración propia con base en los cálculos realizados a partir de la metodología DEA y Malmquist 


\section{Conclusiones}

El índice de productividad de Malmquist muestra un crecimiento de la productividad total de los factores del sistema de transporte de carga internacional por entidad federativa, en donde se refleja el progreso o retroceso en la eficiencia, así como el progreso o retroceso de la frontera tecnológica entre dos períodos de tiempo, 2010 y 2014. Esto, a su vez, permite observar si hay un aumento en la competitividad del comercio exterior mexicano, ya que es altamente significativo, en términos de competitividad, que la mercancía llegue en tiempo y forma al cliente o consumidor.

Se puede concluir, con respecto a los resultados del índice Malmquist, que la mayoría de las entidades federativas han incrementado su productividad en el sistema logístico de carga internacional carretero, para el período de 2010 a 2014. Sin embargo, se aprecia un ritmo de crecimiento muy dispar entre las entidades federativas, esto se hace aún más evidente en la segunda parte del análisis, al interpretar los resultados del cambio tecnológico, donde el diferencial entre la entidad mejor posicionada y la peor posicionada es de 896.75 por ciento.

En cuanto a los resultados arrojados, en el análisis de cambio tecnológico y el cambio en la eficiencia técnica global, tuvieron un comportamiento opuesto, lo que se traduce como una fuerte inversión en algunas entidades federativas, así como innovaciones en los procesos, pero no una adecuada optimización de sus recursos.

Así mismo, el presente trabajo presenta el índice Malmquist desagregado en eficiencia de escala, eficiencia técnica pura, eficiencia técnica global y cambio tecnológico. Lo que permite no solo obtener resultados más precisos, del estado del arte, en materia de logística del sistema carretero de carga internacional, sino que también en este trabajo se hace una aproximación a la propuesta original del modelo de Färe et al. (1994).

El comercio exterior con Canadá y EE.UU, mediante la utilización del transporte carretero, presentó un crecimiento constante a partir de la firma del TLCAN, lo cual puede explicarse por el gran número de maquiladoras estadounidenses establecidas en el territorio mexicano, para las cuales, México otorga facilidades bajo los programas de promoción sectorial, entre otros. Lo que refuerza los resultados del estudio que señalan un incremento en el total de la red carretera que ha mostrado un incremento en el período analizado, aunque en un porcentaje mayor se han incrementado las unidades vehiculares de carga registradas para este sector y, por ende, el personal ocupado. Es 
importante mencionar que el aumento de las unidades vehiculares registrado, no necesariamente significa que las unidades utilizadas para este sector sean unidades nuevas; es decir, un cambio tecnológico exponencial, ya que a partir del 2005 se incrementó la importación de unidades usadas, provenientes de EE.UU.

Por lo que se puede concluir que los estados mejor posicionados o más eficientes son aquellos que han invertido más en las variables utilizadas en el modelo como inputs infraestructura tanto carretera como de infraestructura en las empresas dedicadas al transporte de carga internacional, recursos humanos y calidad. Esto se puede traducir en la necesidad de un esfuerzo conjunto del gobierno y el sector empresarial, a fin de invertir en la mejora de dichos insumos.

Haciendo una reflexión respecto de los resultados de eficiencia obtenidos tras el estudio llevado a cabo por el Banco Interamericano para el Desarrollo en el año 2010, titulado: La logística de cargas en América Latina y el Caribe: una agenda para mejorar su desempeño, se observa como principales resultados, en torno a la logística de carga internacional vía carretera, para el caso de Argentina, que existe un elevado costo logístico donde, entre los principales problemas, destacan los robos del transporte carretero y la falta de calidad en los mecanismos de inspección. Mientras que, Bolivia presenta una escasa capacitación en torno al conocimiento de temas logísticos, así como, la falta de infraestructura y la reducida capacitación profesional de los operadores logísticos. Por su parte, Colombia muestra una baja infraestructura carretera al igual que Paraguay. En ese mismo estudio se señala, para el caso de México, que las carreteras son especialmente críticas en lo referente al comercio exterior, debido al alto porcentaje de flujo de comercio exterior realizado por este medio, y se comenta que el proceso de modernización está aún por completarse. No obstante, puntualiza que el autotransporte es muy eficiente en general, presentando una menor eficiencia en los pasos fronterizos con Estados Unidos. Este documento pone de manifiesto que México mostró una mayor eficiencia que muchos países de América Latina, siendo que apenas marca el inicio del período analizado en el presente trabajo. Lo que abre la posibilidad de realizar otros estudios a nivel países, para observar el cambio tecnológico y niveles de eficiencia comparada a nivel país, y respecto de otros países de América Latina.

Cabe destacar la importancia de realizar estudios del sistema carretero de carga internacional a este nivel de desagregación (entidad federativa), puesto que ello permite conocer no solo los niveles de eficiencia empleados, sino también el nivel de eficiencia de los recursos estatales y federales empleados en términos de infraestructura carretera como impulso al comercio exterior de México. Ya que, actualmente, el principal medio de transporte de las exportaciones 
mexicanas es el transporte carretero, lo que sin duda pone de manifiesto la necesidad de mejorar el sistema logístico carretero de carga si se quiere aumentar o, por lo menos, mantener el posicionamiento de las exportaciones mexicanas con sus socios comerciales y particularmente con Estados Unidos de Norteamérica.

\section{Referencias}

[1] Aghion, P. \& Howitt, P. (1999). Endogenous Growth Theory. Cambridge, Massachusetts: MIT Press.

[2] Aghion, P., Boulanger, J. \& Cohen, E. (2011). "Rethinking industrial policy". Bruegel Policy Brief 2011/04, June 2011.

[3] Aghion, P., Dewatripont, M., Du, L., Harrison, A. \& Legros, P. (2012). Industrial Policy and Competition. NBER, Working Paper No. 18048.

[4] Balassa, B. (1965). "Trade Liberalization and 'Revealed' Comparative Advantage". Manchester School 33, 99-123.

[5] Boschma, R. A. \& Frenken, K. (2006). "Why is economic geography not an evolutionary science? Towards an evolutionary economic geography. Journal of Economic Geography, 11(2), 273-307.

[6] Boschma, R. \& Frenken, K. (2011). "The emerging empirics of evolutionary economic geography. Journal of Economic Geography, 11(2), 295-307.

[7] Boschma, R. \& Martin, R. (2007). "Editorial: Constructing an evolutionary economic geography". Journal of Economic Geography, 7(5), 537-548.

[8] Chávez Martín del Campo, J. C., Mosqueda Chávez, M. T. \& Gómez-Zaldívar, M. (2017). "Economic complexity and regional growth performance: Evidence from the Mexican Economy". The Review of Regional Studies, 201-219.

[9] Chiquiar, D. (2005). "Why Mexico's regional income convergence broke down". Journal of Development Economics, 257-275.

[10] Delgado, M., Porter, M. E. \& Stern, S. (2014). "Clusters, convergence, and economic performance". Research Policy, 1785-1799.

[11] Farole, T. (2011). "Special Economic Zones: What Have We Learned?". The World Bank. Number 64.

[12] Farole, T. \& Akinci, G. (2011). Special economic zones: progress, emerging challenges, and future directions. Washington, DC: The International Bank for Reconstruction and Development/The World Bank.

[13] Frenken, K. \& Boschma, R. A. (2007). "A theoretical framework for evolutionary economic geography: Industrial dynamics and urban growth as a branching process". Journal of Economics Geography, 7(5), 635-649.

[14] gob.mx. (2018a). Zonas Económicas Especiales. Retrieved from Zona Económica de Puerto Chiapas: https://www.gob.mx/zee/articulos/zona-economica-depuerto-chiapas

[15] gob.mx. (2018b). Zonas Económicas Especiales. Retrieved from Zona Económica Especial de Lázaro Cárdenas: https://www.gob.mx/zee/es/articulos/zonaeconomica-de-lazaro-cardenas-la-union?idiom=es 
[16] gob.mx. (2018c). Zonas Económicas Especiales. Retrieved from Zona Económica Especial de Coatzacoalcos: https:/www.gob.mx/zee/es/articulos/zonaeconomica-especial-de-coatzacualcos?idiom $=\mathrm{es}$

[17] Gómez, M. \& Ventosa-Santaularia, D. (2009). "Liberación comercial y convergencia regional del ingreso en México". El Trimestre Económico, vol. LXXVI (1), núm. 301, pp. 2105-235.

[18] Gómez-Zaldívar, F. \& Molina, E. (2018). Zonas Económicas Especiales y su impacto sobre el desarrollo económico regional. Problemas del desarrollo, 193.

[19] Grossman, G. \& Helpman, E. (1991). Innovation and Growth in the Global Economy. Cambridge, Massachusetts: MIT Press.

[20] Guo, Q. \& He, C. (2015). "Production space and regional industrial evolution in China". GeoJournal, 379-396.

[21] Hausmann, R., H Barbero, José (2010). La logística de cargas en América Latina y el Caribe: una agenda para mejorar su desempeño. Banco Interamericano para el Desarrollo (BID). Departamento de Infraestructura y Medio Ambiente. No.IDB-TN-103. Washington, DC. USA, pp. 56-58.

[22] Berg, Sigbjorn Atle; Forsund, Finn y Jansen, Eilev (1992). "Malmquist indices of productivity growth during the deregulation of Norwegian banking, 198089". Scandinavian Journal of Economics 94 pp. 211-228.

[23] Bougheas, Spiros; Demetriades, Panicos O. y Morgenroth, Edgar L. W. (1999). "Infrastructure, transport costs and trade". Journal of International Economics, Elsevier, vol. 47(1), pp. 169-189.

[24] Caplice, Chris y Yossi, Sheffi (2011). "Optimization-based procurement for transportation services". Journal of Business Logistics 24 (2), pp.109-128.

[25] Caves, Douglas; Laurits, Christensen y Erwin, Diewert (1982). "The economic theory of index numbers and the measurement of input, output, and productivity". Econométrica 50(6), pp. 1393-1414.

[26] Cooper, William; Lawrence, Seiford y Kaoru, Tone (2007). Data envelopment analysis. A comprehensive text with models, applications, references and $D E A$-solver software (2nd ed.). Springer.

[27] Cooper, William; Lawrence, Seiford y Kaoru, Tone (2006). Introduction to Data Envelopment Analysis and Its Uses. Nueva York: Springer.

[28] Cornwell, Christopher; Peter, Schmidt y Robin, Sickles (1990). "Production frontiers with cross-sectional and time-series variation in efficiency levels". Journal of Econometrics, Vol. 46, N. ${ }^{\circ} 1-2$, pp. 185-200.

[29] Crainic, Teodor Gabriel y Michael, Florian (2008). "National planning models and instruments". INFOR: Information Systems and Operational Research, 46 (4), pp. 299-308.

[30] Debreu, Gerard (1951). "The coefficient of resource utilization". Econometrica, 19(3), pp. 273-292.

[31] ETAN (2011). Estadísticas de Transporte de América del Norte. (2011). Obtenido de http://nats.sct.gob.mx

[32] Färe, Rolf; Shawna, Grosskopf y Knox, Lovell (1994), Production Frontiers. Cambridge: Cambridge University Press.

[33] Farrell, M. J. (1957), 'The measurement of productive efficiency', Journal of the Royal Statistical Society, Vol. 120, pp.253-290. 
[34] Federal Highway Administration (2005). Logistics costs and the US Gross Domestic Product. MacroSys Research and Technology, Washington, D. C. Department of Transportation

[35] Felipe, Jesus; Utsav, Kumar y Arnelyn, Abdon (2010). "Exports, Capabilities, and Industrial Policy in India.” Working paper no.638. Levy Economics Institute of Bard College, November, pp. 1-34.

[36] Fisher, Ronald Aylmer (1922). "On the mathematical foundations of theoretical statistics". Philosophical Transactions of the Royal Society of London. Series A, Containing Papers of a Mathematical or Physical Character, Vol. 222, The Royal Society, pp. 309-368.

[37] Francois, Joseph and Miriam, Manchin. (2007). "Institutions, Infrastructure, and Trade". Working Paper No. 0705, Johannes Kepler University of Linz, Department of Economics, pp. 1-41.

[38] Flitsch, Verena y Brümmerstedt, Katrin (2015). Freight Transport Modelling of Container Hinterland Supply Chains. Operational Excellence in Logistics and Supply Chains. Agosto Ed. GmbH.

[39] Flitsch, Verena y Brümmerstedt, Katrin (2015b). Cost Functions in Freight Transport Models. Operational Excellence in Logistics and Supply Chains. Agosto Ed. GmbH.

[40] FTA, (2016). Prosperity, productivity, resilience. Delivering Safe, Efficient Sustainable Logistics. Logistics Report. Freight Transport Association. England. UK

[41] Grifell-Tajté, Emili y Knox, Lovell (1993a). "Deregulation and Productivity Decline: The case of Spanish Saving Banks". Working Paper, 93-02 (June), Deparment of Economics, University of North Carolina.

[42] Grifell-Tajté, Emili y Knox, Lovell (1993b). "A New Descomposition of the Malquimist Productivity Index". Working Paper, 93-04 (October) Department of Economics, University of North Carolina.

[43] Grifell-Tajté, Emili y Knox, Lovell (1996). "Deregulation and productivity decline: The case of Spanish saving banks". European Economic Review, 40, pp.1259-1279.

[44] Hertel, Thomas y Tasneem, Mirza (2009). "The Role of Trade Facilitation in South Asian Economic Integration", Chapter 2 The Asian Development Bank Study on Intraregional Trade and Investment in South Asia, Manila: The Asian Development Bank, pp. 12-39.

[45] Hummels, David; Jun, Ishii y Kei-Mu, Yi (2001). "The nature and growth of vertical specialization in world trade". Journal of International Economics, 54 , pp. 75-96.

[46] Lowell, Knox (2002). "Performance Assessment in the Public Sector".

[47] Manheim, Marvin (1979), "Fundamentals of Transportation System Analysis", En Fundamentals of Transportation System Analysis, Vol. 1. MIT press, 1979.

[48] Mankiw, Gregory (2010). Macroeconomics (7th ed.). New York: Worth Publishers.

[49] Malmquist, Sten (1953). Index numbers and indifference surfaces, Trabajos de Estadistica 4, pp. 209-242.

[50] Moorstenn, Hicks (1961). "On Measuring Productive Potential and Relative Efficiency”. Quarterly Journal of Economics 75, pp. 451-467. 
[51] Nishimizu, Mieki y John M. Page (1982). "Total Factor Productivity Growth, Tecnological Progress and Technical Efficiency Change: Dimension of Productivity Change in Yugoslavia, 1965-78". The Economic Journal, 92, no. 368 , pp. 920-936.

[52] Puettman, Carolin y Hartmut, Stadtler (2010). "A collaborative planning approach for intermodal freight transportation". OR Spectrum, 32 (3), pp. 809-830.

[53] Sourdin, Patricia y Richard, Pomfret (2012). "Measuring International Trade Costs". The Word Economy. Volume 35, Issue 6, June, pp.740-756.

[54] Tornqvist, Leo (1936). "The Bank of Finland's Consumption Price Index", Bank of Finland Monthly Bulletin, No. 10, 1-8. 